\title{
Peripheral Resistance to Thyroid Hormone
}

National Cancer Institute

\section{Source}

National Cancer Institute. Peripheral Resistance to Thyroid Hormone. NCI Thesaurus.

Code C131275.

Decreased response to thyroid hormones in peripheral tissues, with normal response in the pituitary gland. 